\title{
Road map to clean energy using laser beam ignition of boron-hydrogen fusion
}

\author{
H. HORA, ${ }^{1}$ S. ELIEZER,,${ }^{2,4}$ G.J. KIRCHHOFF, ${ }^{3}$ N. NISSIM, ${ }^{2,5}$ J.X. WANG,${ }^{6}$ P. LALOUSIS, ${ }^{7}$ Y.X. XU, ${ }^{6}$ \\ G.H. MILEY, ${ }^{8}$ J.M. MARTINEZ-VAL, ${ }^{4}$ W. MCKENZIE, ${ }^{9}$ AND J. KIRCHHOFF ${ }^{9}$ \\ ${ }^{1}$ Department of Theoretical Physics, University of New South Wales, Sydney 2052, Australia \\ ${ }^{2}$ SOREQ Research Centre, Yavne, Israel \\ ${ }^{3}$ UJG Management GmbH, 85586 Poing, Germany \\ ${ }^{4}$ Polytechnique University, Madrid, Spain \\ ${ }^{5}$ Department of Earth and Planetary Science, University of California, Berkeley, CA 94720, USA \\ ${ }^{6}$ State Key Laboratory of Precision Spectroscopy, East China Normal University, Shanghai 200062, China \\ ${ }^{7}$ Institute of Electronic Structure and Laser FORTH, Heraklion, Greece \\ ${ }^{8}$ Department Nuclear, Plasma \& Radiol. Engineering, University of Illinois, Urbana IL, USA \\ ${ }^{9} \mathrm{HB} 11$ Energy Pty. Ltd., Sydney, Australia
}

(Received 26 September 2017; AcCEPTEd 16 October 2017)

\begin{abstract}
With the aim to overcome the problems of climatic changes and rising ocean levels, one option is to produce large-scale sustainable energy by nuclear fusion of hydrogen and other very light nuclei similar to the energy source of the sun. Sixty years of worldwide research for the ignition of the heavy hydrogen isotopes deuterium (D) and tritium (T) have come close to a breakthrough for ignition. The problem with the DT fusion is that generated neutrons are producing radioactive waste. One exception as the ideal clean fusion process - without neutron production - is the fusion of hydrogen $(\mathrm{H})$ with the boron isotope ${ }^{11} \mathrm{~B} 11$ (B11). In this paper, we have mapped out our research based on recent experiments and simulations for a new energy source. We suggest how HB11 fusion for a reactor can be used instead of the DT option. We have mapped out our HB11 fusion in the following way: (i) The acceleration of a plasma block with a laser beam with the power and time duration of the order of 10 petawatts and one picosecond accordingly. (ii) A plasma confinement by a magnetic field of the order of a few kiloteslas created by a second laser beam with a pulse duration of a few nanoseconds (ns). (iii) The highly increased fusion of HB11 relative to present DT fusion is possible due to the alphas avalanche created in this process. (iv) The conversion of the output charged alpha particles directly to electricity. (v) To prove the above ideas, our simulations show for example that 14 milligram HB11 can produce $300 \mathrm{kWh}$ energy if all achieved results are combined for the design of an absolutely clean power reactor producing low-cost energy.
\end{abstract}

Keywords: Clean boron fusion; Laser boron fusion; Non-thermal plasma block acceleration; Petawatt-picosecond laser pulses; Single laser beam ignition

\section{INTRODUCTION}

When the field of nuclear physics was created by Lord Rutherford just after 1900, it was evident from the beginning that the energy exchanges for nuclear processes are about 10 million times higher than those in chemical reactions. The binding energy of the protons and neutrons in a nucleus is of the order of 10 million electron volts $(\mathrm{MeV})$ while the typical chemical reaction binding energies are of about 1 electron volt (eV) only. Using Rutherford's experiments with Albert Einstein's explanation of the connection between

Address correspondence and reprint requests to: Department of Theoretical Physics, University of New South Wales, Sydney 2052, Australia. E-mail: h.hora@unsw.edu.au mass and energy, as demonstrated by the most famous equation in physics $E=m c^{2}$ ( $c$ is speed of light and $E$ is the energy contained in mass $m$ ), it was evident that the burning in nuclear reactions is related to 10 million times more energy than in chemical reactions.

There are two types of nuclear reactions that can yield energy: Fission and fusion. During the fission process, a heavy element like uranium (element number $Z=92$ ) splits into two elements and neutron emission while during the fusion two light elements are to be combined into a heavier one. The nuclear fusion of the light elements, from the one proton hydrogen $(Z=1)$ up to iron $(Z=26)$ can release energies of the order of few $\mathrm{MeV}$ per reaction. The most important nuclear fusion, namely the one that gives life to our planet, is 
the hydrogen cycle producing the helium nuclei $(\alpha)$ and yielding the energy of the sun. This cycle includes the weak interaction of proton-proton fusion creating a deuterium that further collides with a proton to get the helium-3 isotope that fuses with another helium-3 to get the final alpha nuclei.

The proton-proton fusion cross section has by far the lowest cross section of this chain of reactions and it is given by $\sigma(\mathrm{p}+$ $\left.\mathrm{p} \rightarrow \mathrm{D}+\mathrm{e}^{+}+v_{\mathrm{e}}\right)=[S(E) / E] \exp [-2 \pi \eta(E)]$, where $\mathrm{e}^{+}$is the positron and $v_{\mathrm{e}}$ denotes the neutrino (Eliezer \& Ross, 1974) of the electron class. $[S(E) / E]=4.0 \times 10^{-46} \mathrm{keV} . \mathrm{cm}^{2} / E_{\mathrm{keV}}$ and $\eta(E)=2 \pi \mathrm{e}^{2} /(h v)$ where $v$ is the relative velocity between the two colliding protons. The rate and timetable of this cycle are dominated by the $\mathrm{H}-\mathrm{H}$ interaction of the nuclei of the light hydrogen $\mathrm{H}$, the protons.

The fusion power (in watts) is in general given for the interaction of particle 1 with particle 2 by

$$
P[\mathrm{~W}]=n_{1} n_{2}\langle\sigma v\rangle V E[\mathrm{~J}]
$$

The energy power in watts $P[\mathrm{~W}]$ is proportional to the number densities $n_{1}$ and $n_{2}$ times the rate $\langle\sigma v\rangle$, where $\sigma$ is the cross section for fusion and $v$ is the relative velocity between the two fusing particles, times the volume $V$ where the fusion reactions take place, times the average energy released (in Joules) by the fusion reactions $E[\mathrm{~J}]$. For our sun the hydrogen-hydrogen rate of fusion is of the order of $\langle\sigma v\rangle=10^{-43} \mathrm{~cm}^{3} / \mathrm{s}$, an extremely small value. The fusion is possible because the sun is extremely big.

In order to achieve fusion on our planet in a controllable way, one has to reduce the volume by many orders of magnitude and this is possible because we look for strong interaction fusion with a rate $\langle\sigma v\rangle$ of about $10^{-15} \mathrm{~cm}^{3} / \mathrm{s}$, namely many orders of magnitude larger. For this purpose, in the context of energy production on our planet, the fusion (1) of the heavy hydrogen isotopes deuterium (D) and tritium (T) and (2) the hydrogen $\mathrm{H}$ (proton) reaction with the boron isotope 11 (B11) are of great interest

$$
\begin{gathered}
\mathrm{D}+\mathrm{T} \rightarrow{ }^{4} \mathrm{He}+n+17.6 \mathrm{MeV} \\
\mathrm{H}+{ }^{11} \mathrm{~B} \rightarrow 3{ }^{4} \mathrm{He}+8.7 \mathrm{MeV}
\end{gathered}
$$

Using equation (1) for a terrestrial power plant of $P=1$ gigawatt $(\mathrm{GW})$, with an energy output per reaction of about $10 \mathrm{MeV}$ with the maximum reaction rates of $\langle\sigma v\rangle=10^{-15} \mathrm{~cm}^{3} / \mathrm{s}$, we need $n_{1} n_{2} V$ equal about $10^{36} \mathrm{~cm}^{-3}$. There are two distinct possibilities to achieve this limit: (a) A continuous operation with magnetic confinement of dilute plasmas, with the density order of $n=10^{13} \mathrm{~cm}^{-3}$ and large volumes $V=10^{10} \mathrm{~cm}^{3}$ with the confinement of the reacting plasmas by magnetic fields (Bigot, 2017) and - alternatively - (b) Inertial confinement of (a tiny "small sun") compressed solid-state plasma with densities number of $10^{23} \mathrm{~cm}^{-3}$ and larger for pulsating operation.

The discovery of the laser paved the way from the beginning to use it for miniaturization of the ignition for a controlled pulsating fusion energy generation in a controlled way in power reactors (Sakharov, 1960; Basov \& Krokhin et al. 1964; Dawson, 1964; Hora, 1964; Nuckolls, Contributions to the Genesis and Progress of UCF, 2007). The following developments at the National Ignition Facility NIF in Livermore/California with the largest laser in the world arrived at fusion energy gains rather close to but not yet the desired ignition point (Hora, 2013; Hurricane et al., 2014; Hinkel et al., 2016).

For a thermal equilibrium plasma, the DT was the first choice of a fusion reactor because it reaches the high value of $\langle\sigma v\rangle$ of $10^{-15} \mathrm{~cm}^{3} / \mathrm{s}$ for the lowest possible temperature (at $T=20 \mathrm{keV}$ ). In contrast to the DT reaction, the boron reaction HB11 reaction is very much less efficient under conditions of thermal equilibrium. The values of $\langle\sigma v\rangle$ for the clean HB11 fusion is so much lower that the gain is smaller by five orders of magnitude, a factor of 100,000 such this option of fusion was usually excluded. This reaction reaches the large $\langle\sigma v\rangle$ for temperatures at temperatures of about $600 \mathrm{keV}$ and for laser ignition a compression to 100,000 times solid-state density was considered as extremely difficult to be achieved using the conventional theories (see Section 9.6 of Hora, 2016).

The HB11 reaction was of special interest because no neutrons (n) were produced when the reacting H-nuclei (protons) had lower energy than about $3 \mathrm{MeV}$. HB11 is then an environmentally clean reaction in contrast to DT (Tahir \& Hoffmann, 1997). The aim of the neutron-free clean boron fusion was nevertheless highly attractive, that several proposals were developed by highly qualified experts (Meglich \& Morwood et al., 1988). The only way out was to work at conditions without local thermal equilibrium, LTE, and using nonlinearities as concluded from preceding research (Hora, 1988). A further advantage of the HB11 reaction was the generation of three helium nuclei (alpha particles), Eq. (2b), which can be expected for producing an avalanche multiplication.

For fusion at conditions of non-thermal ignition, the laser was the ideal tool when using the result (Hora et al., 2014, $2015 a, b)$ that the electric and magnetic fields $\mathbf{E}$ and $\mathbf{H}$ of a sufficiently intense laser beam cause an acceleration of nearly solid density plasma blocks without heating within a picosecond (ps) interaction to velocities above $10^{9} \mathrm{~cm} / \mathrm{s}$ (Lawrence, 1978; see Figures 10.18a \& b of Hora, 1988). This numerical result of 1978 was exactly reproduced in agreement with the measurements by Sauerbrey (1996) with laser pulses of picoseconds duration and powers between Terawatt (TW) and petawatt (PW) available 1996 and repeated by Földes et al. (2000).

This non-thermal ignition of solid density fusion fuel was the result by Chu (1972) and by Bobin (1974) by initiation of the reaction to be produced within a picosecond, when hydrodynamic computations included - next to thermal properties - the interaction of the laser fields resulting in nonlinear (ponderomotive) forces dominating the generation of the plasma blocks (Hora, 1981, 2016). An updating of the hydrodynamic computations (Hora et al., 2008; Lalousis et al., 2013) and subsequently in full agreement with using 
a more detailed genuine multi-fluid code reproduced these measurements of ultrahigh acceleration for DT-fusion (Sauerbrey, 1996; Földes et al., 2000). Furthermore, it was then a great surprise, when applying this for HB11 fusion (Hora, 2009; Hora et al., 2009, 2010), that the ignition threshold for HB11 was elevated by five orders of magnitudes above the classical values arriving at the level of DT due to the non-thermal transfer of the laser energy directly into the ultrahigh acceleration of the plasma blocks.

Another advantage was the very first measurement of HB11 fusion (Belyaev et al., 2005) by using lasers with thousand reactions per laser shot, followed by more than 1 million reactions (Labaune et al., 2013) and then by one billion reactions (Picciotto et al., 2014). Comparison with DT fusion confirmed that this could be achieved only by avalanche reactions (Hora, 2014; Hora et al., 2015). The detailed evaluation of these measurements (Margarone et al., 2015) based on elastic nuclear collisions towards the exceptionally high HB11 reaction around $600 \mathrm{keV}$ (Eliezer et al., 2016; Hora et al., 2017b) showed an increase of the gains by further four orders of magnitudes.

Based on these nine orders of magnitudes higher reactivity of HB11 at non-local thermodynamic equilibrium (LTE) conditions, a fusion power reactor was designed (Hora et al., 2014; Lalousis et al., 2014; Hora, 2015) with picosecond laser pulses of more than 30 Petawatt power that are expected to ignite 14 milligram hydrogen-boron for producing $300 \mathrm{kWh}$ energy.

The following sections describe properties for aiming environmentally clean, low cost, and sustainable energy production, and a list of research topics. Single laser beam ignition for fusion energy is an innovation for alternative conditions differing from the usual spherical irradiation by many synchronized laser beams. These developments were initiated in 2014 (Hora et al., 2014, 2015a; Lalousis et al., 2014) following the Patent Cooperation Treaty international patent with the priority of 23 March 2014 (Hora \& Kirchoff, 2017) for the now progressing national phases (Hora et al., 2017a). These developments (Hora et al., 2015; Hora \& Kirchhoff, 2015a) followed step by step (Hora et al. $2017 b, c$ ) for the first realistic option of an environmentally absolute clean reactor for the fusion of hydrogen with the boron isotope 11 (HB11) for low cost and sustainable largescale energy generation.

HB11 fusion (Oliphant \& Rutherford, 1933) is well known to avoid generation of neutrons that in all other cases of nuclear energy reactors are producing dangerous radioactive waste. The energy of the reacting hydrogen nuclei, the protons, have to have energies below $\mathrm{MeV}$ for a reaction completely free from neutrons.

The disadvantage of HB11 fusion is that it is about five orders of magnitudes - 100,000 times - more difficult and was considered as impossible (see Chapter 9.6 of Hora, 2016) compared with the easiest fusion of heavy with superheavy hydrogen, deuterium and tritium at conditions of LTE of the reacting plasma. And even DT fusion is still only close below breakeven despite enormous research using magnetic confinement (MCF) (Bigot, 2017) or inertial confinement (ICF) (Hora, 2013) fusion.

Against these facts of the impossibility of HB11, the aneutronic aspect attracted prominent experts to search for solutions (Hora et al., 2017a). This in principle could only be based on conditions without thermal equilibrium (Hora, 1988; Hora et al., 2012) and without using nonlinear effects (see clarifications by Feynman, Section 6.3 of Hora, 2016). An example of studies of plasmas different from LTE thermal equilibrium plasmas was developed for the dusty plasmas (Fortov \& Iakubov, 1998) with similar problems to the HB11 fusion (Hoffmann et al., 2017) or non-neutral plasmas (Davidson, 2016). Attempts are using low-density plasma (Hirsch, 1972; Rostoker et al., 1997; Miley \& Murali, 2015) where however no fusion reactions were measured jet (Hora et al., 2017a). Following the principle (Hora, 1988; Hora et al., 2002) to use a non-thermal ignition of HB11 fusion by laser pulses led to measured high gain HB11 reactions (Hora et al., 2015) opening the option that ultra-highly accelerated plasma blocks with conversion of laser energy directly into directed plasma block motion in the range of solid-state densities, the present achievement is a nine orders higher gain of fusion energy than classical measured (Eliezer et al., 2016). This is even higher than DT (Hora et al., 2015). The reason is the non-thermal ignition and the avalanche multiplication (Hora et al., 2014) of the generated three helium nuclei of reaction in agreement with the theory (Eliezer et al. 2016) for the experiments (Picciotto et al., 2014; Margarone et al., 2015).

\section{LASER BORON FUSION REACTOR WITH BASICALLY NEW PROPERTIES}

Experiments for laser-driven DT fusion were based on spherical irradiation of laser beams. The laser amplifiers of the NIF experiment cover most of the size of three football fields for the experiment where the beams are collected by a $10 \mathrm{~m}$ diameter sphere (Fig. 1) to be focused into the center. The techniques of correct guiding, focusing and temporal scheduling of 196 laser beams were developed.

For the laser boron fusion, the reactor is of spherical shape, Figure 2, but with the basic simplification that the ignition of the reaction is produced only by one laser beam. The wall of the reactor sphere of at least $1 \mathrm{~m}$ radius for sustaining the generated helium nuclei (alpha particles of $2.9 \mathrm{MeV}$ energy) of $300 \mathrm{kWh}$ energy per shot has to be of steel or similar material of at least view millimeter thickness. The shock produced by the fusion reaction corresponds to that of a chemical explosive of about $50 \mathrm{~g}$ (Hora et al., 2015). This comparably low shock compared with chemical reactions is due to the fact that this depends on the energy of the generated particles. This is given by the square root of the ratio between nuclear and chemical energy, reducing the nuclear explosion shock by a factor of few thousands against the 


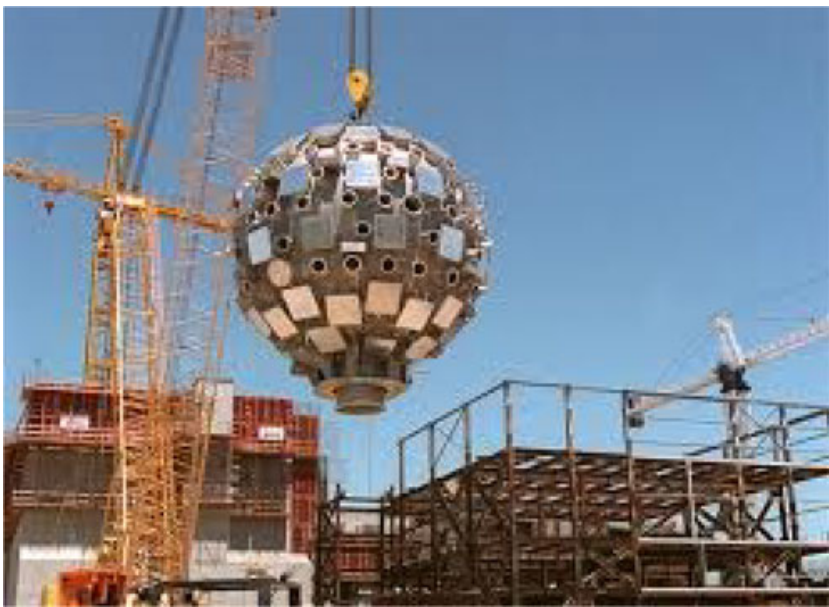

Fig. 1. Sphere of $10 \mathrm{~m}$ diameter at the NIF laser at the Lawrence Livermore National Laboratory LLNL in California where Laser beams are irradiated into the holes to and focused to diameters of about 100 micrometer diameter into holes of a capsule for indirect drive DT fusion in a sphere with a diameter above one millimeter producing the highest nuclear fusion gains (Hurricane et al., 2014; Hinkel et al., 2016).

chemical explosion apart from a softening to the shock front of the alphas.

The basic difference to the reaction unit in the center of the sphere is that this is not for a spherical irradiation but for a cylindrical geometry of the fusion fuel Figure 3 about which details are to be discussed in the following section. If the unit is at the same potential as the sphere, the energy of the alphas is absorbed in the wall sphere and has then to be converted thermally for use in electric generators.

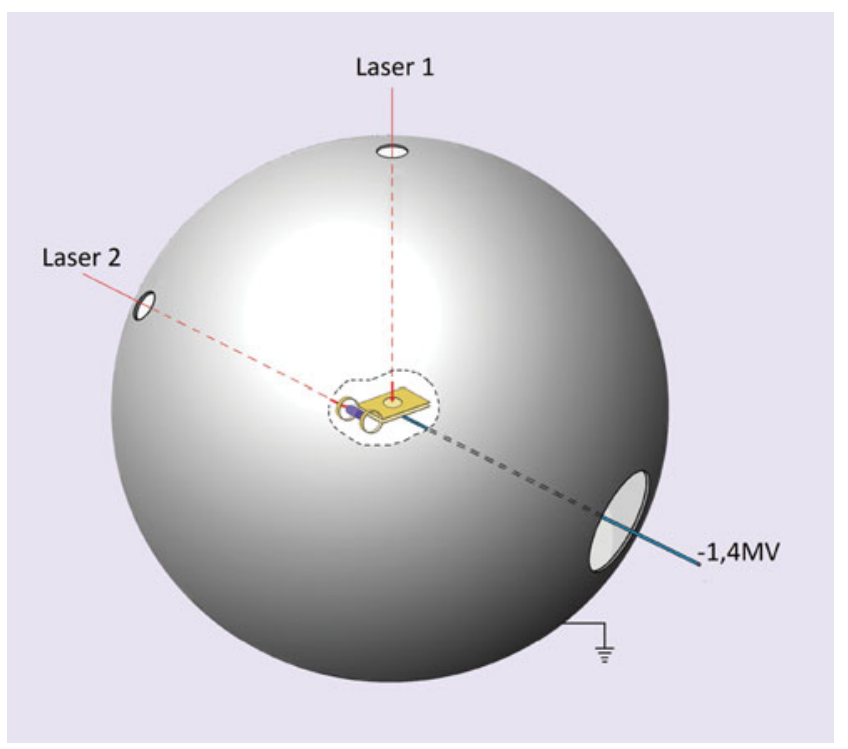

Fig. 2. Scheme of an economic electric power reactor for production of boron-fusion, absolutely free from the problem of dangerous nuclear radiation (Hora et al., 2014, 2015a, b) with the estimated possibility of a power station producing electricity of up to a profit of $\$ 300$ million/year (Hora, 2015; Hora et al., 2017b). Description of central reaction unit, see Figure 3.

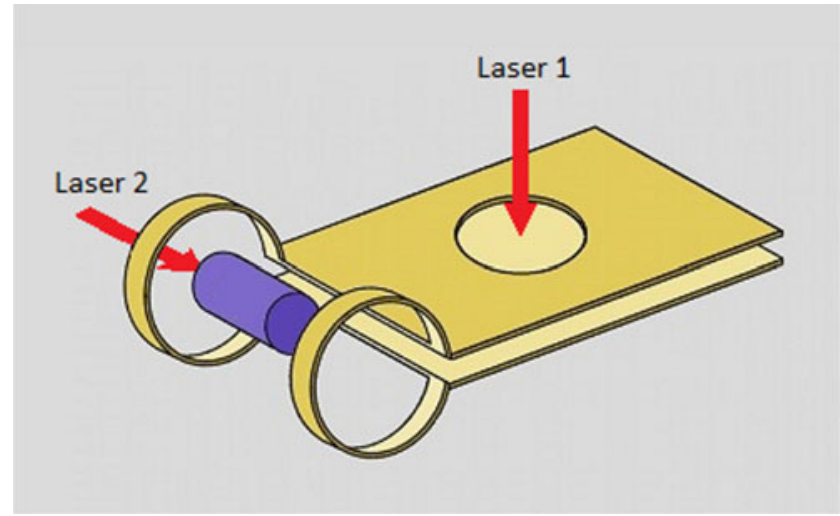

Fig. 3. Reaction unit in the center of the reactor of Figure 2 using "capacitor coil fields" (Fujioka et al., 2013). The cylindrical target with the HB11 fuel is co-axially located in a coil where during a ns a 4.5 kilotesla magnetic field is produced by a $\mathrm{kJ}-\mathrm{ns}$ pulse of laser 1 (Fujioka et al. 2013; Santos et al., 2015; Tikhonchuk et al., 2017) for trapping the fuel plasmas. A ps-30 kJ laser pulse 2 initiates the non-thermal ignition of the fusion in the fuel (Hora, 1988; Hora et al., 2015, 2017c).

Another advantage is that nuclear energy of the monoenergetic alphas can be changed directly into electricity with a minimum of thermal losses into cooling systems if the unit is charged on a negative potential of less than but close to $1.4 \mathrm{~V}$. The alphas are then slowed down when flying against the positive wall potential and the electrostatic gained energy can then directly converted into three-phase electric currents by techniques well developed by the HVDC high-voltage direct current transmission lines (Kanngiesser et al., 1994; Hammons et al., 2012). This direct conversion of nuclear energy into electricity is indeed possible only if plasma discharge losses between the unit equipped with Faraday screening and the reactor wall can be sufficiently reduced, otherwise, the energy conversion of the alphas is possible only by the heating of the wall material.

What else is standard level for use in the HB11-reactor of Figure 2? Lasers for pulses up to picosecond duration and more than $30 \mathrm{PW}$ power are well expectable within few years. In 2017, lasers with $0.17 \mathrm{ps}, 10 \mathrm{PW}$ power and one shot per minute are in use following Todd Ditmire (2017) and upgrading to the other specifications with one shot per second should be developed within the range of present-day technology, driven also for other most important applications than for laser boron fusion. The optical technology for guiding the $30 \mathrm{PW}-$ ps of high contrast and modest focusing is on a much higher level developed for the case of NIF (Fig. 1) can be taken over, well appreciated that the achieved level at NIF is on a breathtaking high level.

The physics of the generation of the ultrahigh magnetic fields in the coils (Fujioka et al., 2013) is indeed an outstanding achievement following the work of Daido et al. (1986) and of Hohenberger et al. (2012) using nanosecond laser pulses of usual level. Nevertheless, the studying of the field properties, the time dependence, and further improvement are of technology for laboratory projects on usual level. 
The mechanically guiding of the reaction unit to the rector center is indeed not an easy topic but should be less difficult than the similar problems in the technology of micro-electronics and may be taken over from there. The technology for repeating the positioning of the unit into the reactor center for one event per second were following solutions based on technologies envisaged by Erhard Gaul (2017).

\section{CYLINDER TARGET PROPERTIES FOR A LASER BORON FUSION REACTOR}

Laser ignition of fusion in a cylindrical fuel target is in indifference to the most studied spherical ignition (Hora, 1964; Hora et al., 1998, and see Chapter 9.4 of Hora, 2016) with adiabatic plasma compression and thermal ignition. For cylindrical ignition, the studies of plane laser waves at normal incidence on a plane plasma target are of interest in one dimension. The computations are including the forces from the gasdynamic plasma pressure but on top, there is a further pressure from the electrodynamic laser field which can dominate over the thermal forces if the laser has a sufficiently high intensity. Figure 4 is the result where a deuterium plasma layer with critical density at $x=0$ density not far from the solid state is irradiated by a neodymium glass laser with $10^{18} \mathrm{~W} / \mathrm{cm}^{2}$ intensity from the right-hand side. The fully drawn line is the density of the laser energy given by the electric $E$ and magnetic $B$ field of the laser. The intensity at the vacuum at the right side end is increased in the plasma due to the optical constant like in glass resulting in an increase to a maximum, and into a deeper decay due to absorption similar to metallic absorption of light. The negative gradient of this curve produces a nonlinear (or

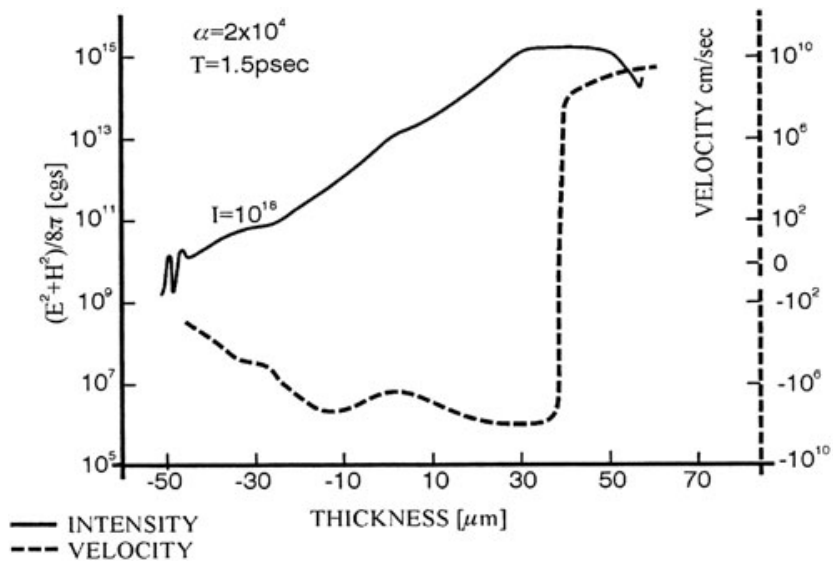

Fig. 4. An intensity of $10^{18} \mathrm{~W} / \mathrm{cm}^{2}$ neodymium glass laser incident from the right-hand side on an initially $100 \mathrm{eV}$ hot very low reflecting deuterium plasma profile produces after 1.5 ps two non-thermally driven plasma blocks by the non-linear (ponderomotive) force (Lawrence, 1978), one block moving against the laser irradiated from the right-hand side and another block moving into the target interior (Fig. 8.4 of Hora, 2016). The laser intensity is expressed by the energy density of the laser field $\left(\mathbf{E}^{2}+\mathbf{H}^{2}\right) / 8 \pi$ electric $\mathbf{E}$ and magnetic field $\mathbf{H}$. ponderomotive) force in the plasma which is much larger than the gasdynamic force for an initial plasma temperature of about 1 million degree and despite further heating by the laser that all is carefully included in the computation. $1.5 \mathrm{ps}$ after the laser interaction the non-thermal laser force accelerated the plasma to the velocities shown in the dashed line. There is a plasma block moving with a velocity above $10^{9} \mathrm{~cm} / \mathrm{s}$ against the laser light in the outer part and another block (negative velocity) moving into the deeper part of the deuterium. The acceleration for these blocks has the astronomical value above $10^{20} \mathrm{~cm} / \mathrm{s}^{2}$.

The result of Figure 4 was from computations in 1978 (Lawrence, 1978; Hora, 1981). The necessary picosecond laser pulses of the power of up to Petawatt were available only in 1996. The measurement of the plasma blocks was then possible, Figure 5 by Sauerbrey (1996) from the Doppler blue shift of spectral lines in the reflected light arriving at the theoretically predicted value of the ultrahigh acceleration above $10^{20} \mathrm{~cm} / \mathrm{s}^{2}$. This was about 100,000 times higher than any acceleration measured in a laboratory. The experiment was not easy to be reproduced but well repeated soon by Földes et al. (2000).

The nonlinear force-driven ultrahigh accelerated plasma blocks had a further extreme property. The space-charge neutral plasma blocks had ion current densities more than million times higher than any classical ion accelerator could produce. It was then possible that the picoseconds-Petawatt laser pulses produced the ultrahigh density accelerated ion pulses of few to several hundred $\mathrm{MeV}$ ions. This was measured in agreement with complicated computations. Interestingly the acceleration could be calculated in a transparent and understandable way by the genuine multi-fluid hydrodynamic models (Banati et al., 2014) needed for hadron cancer therapy. This completed hydrodynamics demonstrated for the first time directly (Lalousis \& Hora, 1983; Hora et al., 1984; Eliezer et al., 2014), how inhomogeneous plasmas have very high internally electric fields and their dynamic changes.

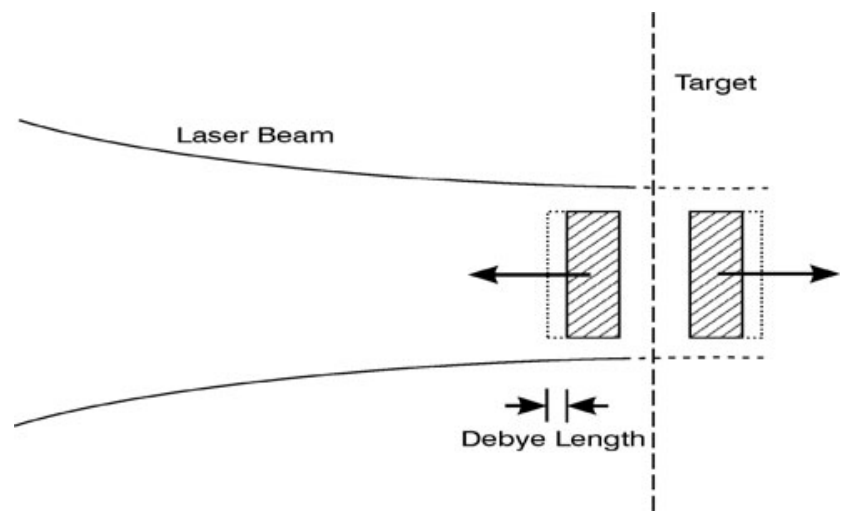

Fig. 5. Ultrahigh acceleration of plasma blocks by the dielectric explosion of laser pulses of picoseconds duration calculated in 1978 by non-thermal forces of laser interaction and measured by Sauerbrey (1996) as predicted (Hora, 1981). 
For ignition of laser fusion, the plasma blocks were needed for the first general use of the initiation of fusion reactions by picoseconds laser pulses irradiating uncompressed, solidstate fuel. Chu (1972) and Bobin (1974) evaluated the laser properties for igniting uncompressed solid density fusion fuel. The result was that the initiation of a fusion reaction needed an impact of an energy flux of $4 \times 10^{8} \mathrm{~J} / \mathrm{cm}^{2}$ during one picosecond as a threshold for generating a fusion flame in DT. For the computation, the above described nonlinear force driven plasma block generation could then be used and the later known plasma phenomena had to be included for reproducing the ignition of DT (Hora et al., 2008). It was then a surprise when using the fusion cross sections of HB11 were used in the calculations: The thresholds for B11 were nearly the same (Hora, 2009; Hora et al. 2010) as for DT. Fusion ignition of HB11 was then happening at five orders of magnitudes higher thresholds than the classical HB11 fusion at thermal equilibrium. These computations used the single fluid hydrodynamics as Chu (1972). Using the genuine two-fluid code (Lalousis \& Hora, 1983; Hora et al., 1984) arrived at the same ignition but with many more details of shock generation and thermal plasma effects beyond the analytical result of the Rankine-Hugoniot theory.

The reaction rate in solid state HB11 fuel depending on the depth $x$ of the fuel at different times is shown in a case of Fig. 6. The reaction front moves with a velocity in the range of $1000 \mathrm{~km} / \mathrm{s}$ which value is similar as known from explosive nuclear reactions. It is a remarkable generation of the shock density profile as it is building up very much later than the ps plasma block initiation of the reaction. This delay is $500 \mathrm{ps}$ and more.

The one-dimensional (1D) conditions were nearly sufficiently well given in the experiments of Sauerbrey (1996)

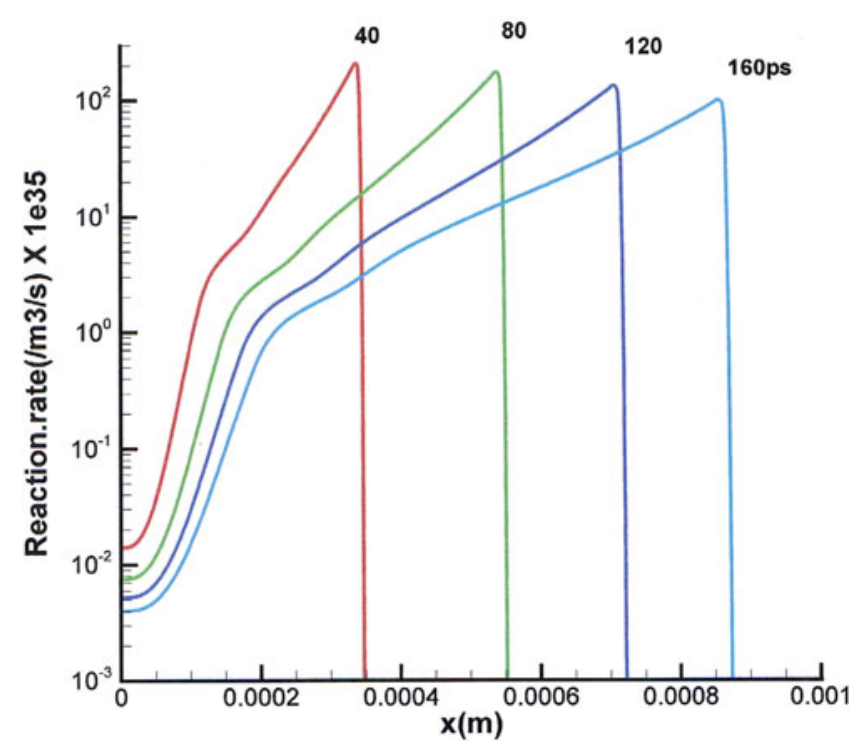

Fig. 6. HB11 fusion reaction rate at different depths $x$ in 1D computation at times after the ps generation of the fusion flame initiated by a $10^{20} \mathrm{~W} / \mathrm{cm}^{2}$, ps, 248-nm wavelength laser pulse. or later for clearly demonstrating that the generated plasma blocks were generated in the skin depth of the irradiated plasma (Badziak et al., 1999; Hora et al., 2002, 2007; Hora, 2003) as seen from the unchanged numbers of the ion in the fast blocks at varying laser intensities at the same focusing. In order to take care of the restricted laser beam diameter and to produce a cylindrical geometry of the reaction as shown in Figure 3, the trapping of the reaction at a constant radius is necessary. This can be done by the ultrahigh magnetic field by the laser produced plasma with the plates as shown in Figure 3. Figure 7 shows the result of trapping of the plasmas to the initial radius of $1 \mathrm{~mm}$ by a magnetic field of 5 kilotesla is evident. The number of the generated alpha particles is shown at different times. The radial trapping at different times is confirmed and increasing on time indicating the successful ignition.

Parallel to these developments with computations of binary fusion reactions for HB11 similar to the computations using DT, the measurements of highly increased fusion gains of with boron (Picciotto et al. 2014; Margarone et al. 2015) was not only clarified by comparison with gains from DT for confirming the avalanche multiplication (Hora et al., 2014, $2015 a, b$ ) of generating each three alpha particles per reaction. Evaluating the experiments (Margarone et al., 2015) by elastic collisions within the extremely non-equilibrium plasma confirmed the avalanche process in all details (Eliezer et al., 2016), Figure 8.

Summarizing all the mentioned processes permitted the computation (Lalousis et al., 2014) that the single beam direct drive HB11 fusion in the cylindrically trapped fusion plasma with end-on direct drive plasma block ignition will produce a very high energy gain. The final result for a power reactor of Figure 2 (Hora, 2015; Hora et al., 2014, $2015 a, b)$ will then result in the reaction of 14 milligram boron-11 with the protons of hydrogen generating more

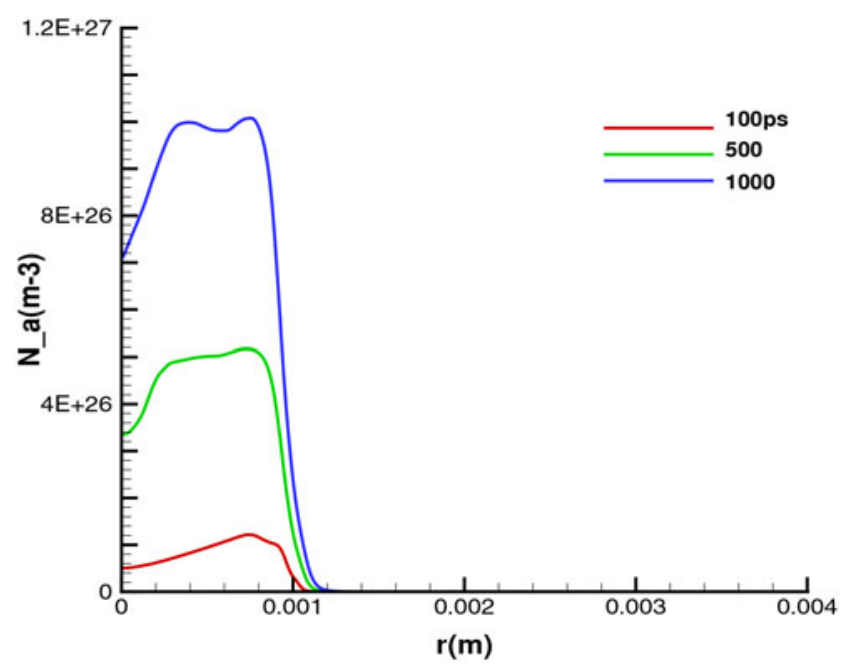

Fig. 7. Radial trapping of the plasma in a cylindrical reaction volume of Figure 3 where the density of the generated alpha particles, N_a, is shown at different times after the block ignition by the ps laser pulse. 


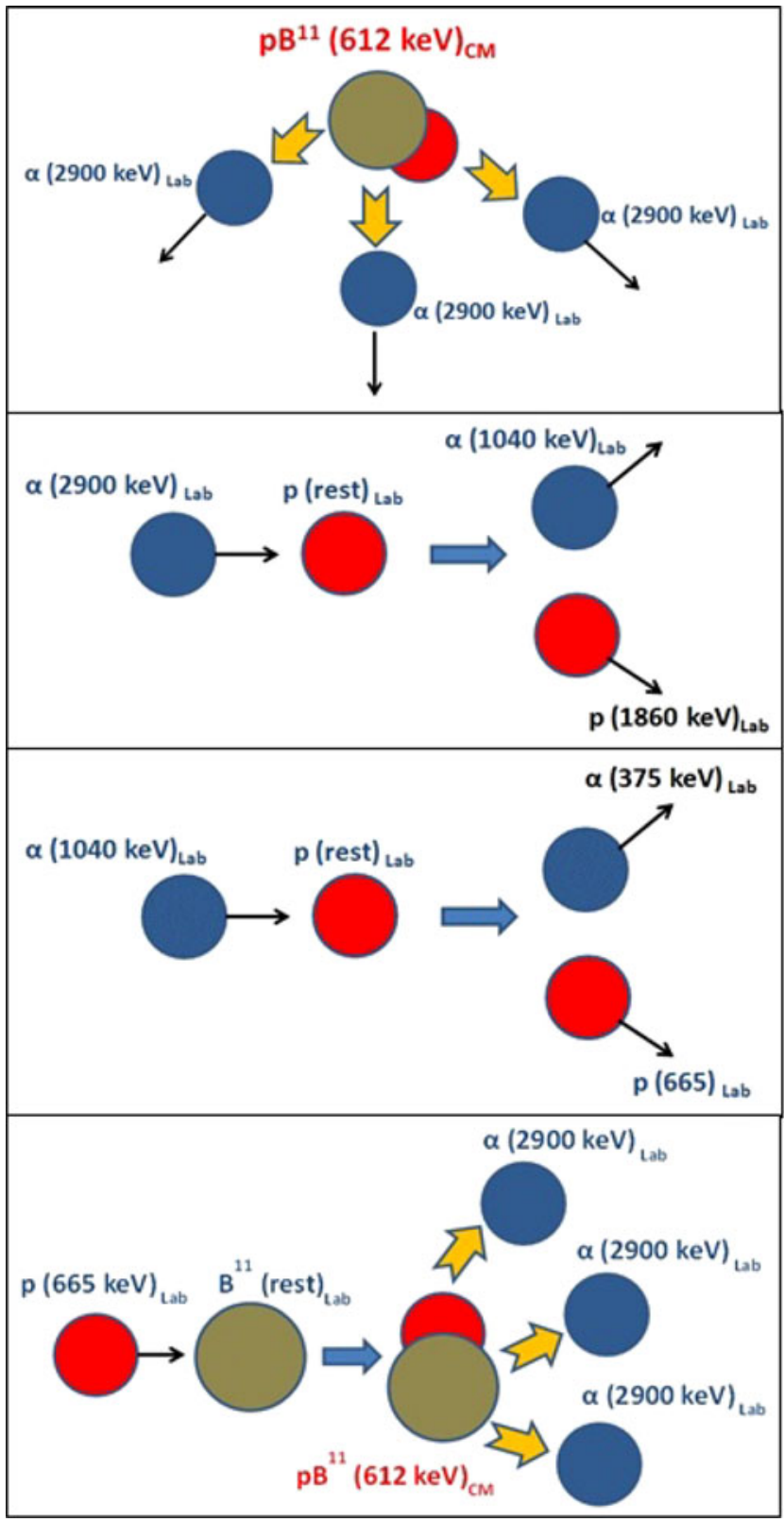

Fig. 8. The three alpha particles of same energy produced by the HB11 reaction, Eq. (1), transfer their energy by elastic collisions with the lowenergy boron or hydrogen nuclei of low temperature in the thermal nonequilibrium background plasmas (Eliezer et al., 2016).

than one Gigajoule $(\mathrm{GJ}=277 \mathrm{kWh})$ energy. The single laser beam for the ignition has to be a picoseconds laser pulse of $30 \mathrm{~kJ}$ energy of $30 \mathrm{PW}$ power, which kind of pulses are rather close to the state of the art if needed one shot per second for energy generation of the value of more than $\$ 100$ million/year (Hora et al., 2017b, c).

\section{PROBLEMS TO BE SOLVED}

The open problems for the single beam cylindrical HB11 fusion reaction are the detailed mechanisms in the ignited cylindrical reaction plasma. The basic step is the combination of the well-developed non-thermal equilibrium initiation of the reaction blocks driven by forces of the laser-plasma interaction, together with the new trapping kilotesla magnetic fields as the key process (Fujioka et al. 2013; Hora et al. 2014, 2017b). The plasma block ignition is fully explored based on the initial fusion flame generation (Chu, 1972; Bobin, 1974; Hora et al., 2008) using ultrahigh acceleration of plasma layers as measured from blue-shifted spectral lines (Sauerbrey, 1996; Földes et al., 2000) is in exact agreement with theoretical predictions (Hora, 1981; Hora, 2016; Hora et al., 2007, 2008). The trapping of cylindrical targets by more than kilotesla magnetic fields was shown (Lalousis et al., 2014; Hora, 2016) (Fig. 7) by computation under temporally constant conditions of the magnetic field.

In reality, the capacitor magnetic coil fields in Figure 3, are not constant and have a complicated spatial and temporal development while the fusion reaction process in the fuel - well evaluated for 1D geometry - will be modified. This flexibility will indeed give the chances for well advantageous conditions for selecting the best time frames for scheduling the block ignition of laser 2 with the constant timing of the laser 1 for operating the capacitor coil of Figure 3. These conditions have to be studied well with techniques available in established laboratories with sophisticated technologies parallel to specific computations using hydrodynamics or particle-in-cell PIC methods. The knowledge gained from this research will lead to generalizations of the properties of the laser pulse 1 with respect to focusing, time dependence and the incidence angle of the beam on the capacitor plates.

The extreme laser pulse 2 has, first of all, to be generated with a very perfectly controlled suppression of pre-pulses. Though these pulses are normally of 1 ps duration or shorter, their temporal pulse shape can well be modified by now available control. This also will give some variability for the temporal scheduling in view of magnetic field development under the control of Laser 1. These are rather sophisticated experimental tasks but are not new and have been solved before.

Another possibility to optimize the conditions for the fusion process of the conditions of the fuel may be if the magnetic field will not only be cylindrical symmetric geometry but may get an additional conical component by modifying the coils in Figure 3 in an asymmetric way with respect to the axis.

While studying the mentioned parameters, there is one question, how the fuel in Figure 3 is behaving within the ultrahigh magnetic fields, even without irradiation of the pulse from laser 2 for the fusion ignition. Under the growing magnetic field in the coil, it will be interesting to find out how the fuel substance - or any other material - will behave under the extreme magnetic fields before reaching the range of kilotesla and more. Will an ionization happen and under what magnetic field strengths? This kind of magnetic field ionization is a topic that may never have been studied before. If a plasma would be generated, or how this may be 
controllable by providing or reducing a certain electric conductivity in the fuel under the extreme conditions, this all may be a further new parameters that can be developed for optimizing the conditions of the new type of cylindrical fusion reactions in Figures 2 and 3 differing from the highly explored fusion in spherical fuel targets.

Progress in this research - but not as a condition of high priority or necessity - is the new direction to study the non-LTE conditions in plasmas as a basically new task for theory. The long desire of the environmentally clean HB11 reaction (excluding exceptional cases of higher energies for nuclear reactions) is of such high interest that all attempts are to be considered to achieve this, under the conditions of non-thermal equilibrium and using nonlinearities (Hora, 1988). These conditions are best given by lasers using high plasma densities near the solid state and are fulfilled with the highest laser-fusion gains measured (Picciotto et al., 2014), repeated and even increased by a factor 10 (Giuffrida et al., 2017) and directly visible from the analysis using elastic nuclear collisions (Eliezer et al., 2016; Hora et al., 2017b, $c)$. A very important new approach is the result of proving these non-equilibrium properties by theoretical evaluations of NIF-experiments (Fan et al., 2017).

The research for low density plasmas for HB11 reactions (Hora et al., 2017a) is well under way, especially with the Tri-Alpha-Energy project (Rostoker \& Binderbauer, 1996) of which the reverse magnetic field design is a modification of the cylindrical version of the Hirsch-Miley configuration of inertial electrostatic confinement IEC (see Section 2 of Hora et al., 2017a). With all these configurations, not any HB11 fusion was measured yet in these low-density plasmas, while the laser-experiments (Belyaev et al., 2005; Labaune et al., 2013; Picciotto et al., 2014) arrived at the super-high reaction gains above those from DT.

The extensive studies of plasmas since 1940 have gained an enormous amount of results with very numerous, unexpected discoveries where the new developing work on HB11 fusion is only one of the subdivisions. How this exploration of science is crucial for the enormous problems ahead, was very well envisioned by the formulations of the problems of plasmas by one of its greatest masters, Edward Teller, on the background also of the complex problems discovered and pioneered by Lord May of Oxford (Robert M. May) as fundamental orientation (see pages 2-4 of Hora, 2016).

\section{NEED FOR VERY HIGH CONTRAST RATIO OF PW-PS LASER PULSES}

The experiments for the studies listed in the preceding section may be performed with lasers of the usual quality with PW-ps pulses. However, very extreme quality is necessary with respect to the contrast ratio for measuring the single beam initiation of the ignition process, characterized by the blue Doppler shift in the reflected light. These high-class laser beams were well available, but only in the very rare cases of with the KrF lasers used by Sauerbrey (1996) and
Földes et al. (2000) and by the extremely precisely developed solid-state lasers used by Zhang et al. (1998) and by Badziak et al. (1999). When Sauerbrey (1996) tried to repeat the measurements at Rutherford Appleton Laboratory with much more powerful $\mathrm{KrF}$ lasers in cooperation with many top class physicists, the results could not be reproduced. The same happened when Badziak was invited to use the solid-state lasers at the Ecole Polytechnique in Paris, the pioneering results of (Badziak et al., 1999) this could not be reproduced. The reason was very evident: The lasers for the reproduction were not good enough!

For using the single beam ignition of the HB11 fusion, see Figures 2 and 3 in difference from multi-beams for spherical irradiation, Figure 1, the fusion fuel is not spherical (Hora et al., 1998), but of cylindrical geometry, Figure 5, with trapping the reaction plasma by ultrahigh magnetic fields (Fujioka et al., 2013) of several kilotesla. Use is then made from the extensively studied non-thermal driving of plasma bocks by the nonlinear force, Figure 4 [see Fig. 8.4 from Hora (2016) drawn from Figs. 10.18a \& b of Hora (1981)]. The $10^{5}$ times higher acceleration than ever measured before in a laboratory for interacting within pico-second laser pulses giving velocities of more than $1000 \mathrm{~km} / \mathrm{s}$, was measured from the blue Doppler-shift of reflected spectral lines (Sauerbrey, 1996; Földes et al., 2000). This blue-shift is in exact agreement with the theoretical predictions from the hydrodynamic computations (Hora, 1981; Hora et al., 2007).

The cylindrical fusion fuel is shown in the axis of the coils of Figure 3 and the irradiation by the single igniting laser pulse is shown end-on at the cylinder of the fuel. The details of the description of the HB11 reactor are based on extensive results of the plane-wave plasma-block generation with detailed experimental and theoretical confirmation. It should be noticed that the new HB11 clean fusion reactor may be listed under the category of fast ignition by Tabak et al. (1994) but it is essentially different from usual schemes as no plasma compression etc. is needed for the new HB11 case.

For designing the next following experimental confirmation of the ignition processes by $>$ PW-ps laser pulses it is necessary to underline the required extremely high contrast ratio of these laser pulses. This contrast has been verified in the preceding experiments and can be taken over from there, but this needed very sophisticated laser technology. It has been achieved in the experiments with $\mathrm{KrF}$ excimer lasers (Sauerbrey, 1996) and only the most exceptional attention and experience did clarify the conditions for excluding relativistic self-focusing by using solid-state lasers (Zhang et al., 1998) convincingly demonstrated by Jie Zhang. It is important to note that the solid-state lasers used in this extremely high contrast were fulfilled in the unique experiments showing the nonlinear-force driven plasma-block acceleration directly from the skin-layer acceleration (Hora et al., 2002; Hora, 2003). Repeating these experiments in other countries by the same experts did fail due to the insufficient quality of the used lasers with respect to contrast properties. This insufficient quality of the usual laser pulses can 
be seen from the broad stream experiments resulting in a red Doppler-shift instead of the blue shift. This could be understood by using PIC computations with plasma densities below critical. The very difficult calculations at critical density were mastered only by JW Wang and his team recently (Xu et al., 2016, 2017; Li et al., 2017; Hora et al., 2017c).

As a test whether the PW-ps laser pulses have a sufficient contrast ratio may be that the light reflected from the irradiated target has to show a blue Doppler shift of spectral lines. Any other lasers cannot be used for studying the non-thermal plasma block ignition at the direct drive on the cylindrical end of theHB11 fuel. When checking earlier experiments, it was elaborated (Hora, 2003) that the extremely unique laser-fusion experiments by Norreys et al. (1998) and Clark et al. (2001) were so exceptional and significant because there seemed to be at least for a short period, laser pulses available with the extreme contrast conditions. When re-evaluating the fusion gain with a 100:1 increase when estimating the DT value from $\mathrm{DD}$, the absolute record gain of about 0.3 was achieved. This is the highest than ever produced gains by laser driven fusion and the highest gains ever reported from tokamak experiments (see p. 218 of Hora, 2016).

\section{ACKNOWLEDGMENTS}

The authors involved with the here given elaboration for the next steps for a laser boron fusion reactor gratefully acknowledge the work of the authors in the cited preceding contributions, especially to L. Giuffrida et al. (2017) for guiding the cooperation by Prof. Georg Korn, Prof. Karel Jungwirth and Dr. Jiri Ullschmied at the European ELI-Beams Project and the PALS Laser of the Academy of Science of the Czech Republic in Prague.

\section{REFERENCES}

Badziak, J., Kozlov, A.A., Makowski, J., Paris, P. \& Ryc, L. (1999). Investigations of ion streams emitted from plasma produced with a highpower picosecond laser. Laser Part. Beams 17, 323.

Banati, R., Hora, H., Lalousis, P. \& Moustaizis, S. (2014). Ultrahigh laser acceleration of plasma blocks with ultrahigh ion density for fusion and hadron therapy. J. Intense Pulsed Laser Appl. Adv. Phys. 4, 11-16.

Basov, N.G. \& Krokhin, O.N. (1964). In Proceedings of 3rd International Quantum Electronics Conference Paris 1963, (Grivet, P. and Bloembergen, N., Eds.), Vol. 2, p. 1375. Paris: Dunod.

Belyaev, V.S., Matafonov, A.P., Vinogradov, V.I., Krainov, V.P., Lisitsa, V.S., Roussetski, A.S., Ignatyev, G.N. \& Andrianov, V.P. (2005). Observation of neutronless fusion reactions in picoseconds laser plasmas. Phys. Rev. E 72, 026406.

BIGOT, B. Key Lecture at the IEEE SOFE conference Shanghai/ China 4 June 2017.

BobIN, J.-L. (1974). Nuclear fusion reactions in fronts propagating in solid DT. In Laser Interaction and Related Plasma Phenomena, Schwarz, H. and Hora, H., Eds.), Vol. 4B, p. 465. New York: Plenum Press.

Chu, M.S. (1972). Thermonuclear reactions waves at high densities. Phys. Fluids 15, 412
Clark, E.L., Krushelnik, K., Zepf, M., Tataralis, M., Machacek, M., Santla, M.I.K., Watts, I., Norreys, P.A. \& Dangor, A.E. (2001) Energetic Heavy-Ion and Proton Generation from Ultraintense Laser-Plasma Interactions with Solids. Phys. Rev. Letters 85, 1654.

Daido, H., Miki, F., Mima, K., FujtTa, M., Sawaki, K., Fujita, H., Kitagawa, Y., NaKaI, S. \& YamanaKa, C. (1986). Generation of a strong magnetic field by an intense CO2-laser pulse. Phys. Rev. Lett. 56, 846.

Davidson, R. (2016) Nonneutral Plasmas. Singapore: World Scientific.

Dawson, J.M. (1964) On the production of plasma by giant pulse lasers. Phys. Fluids 7, 981.

Ditmire, T. (2017) Proceedings SPIE Conference 10241 paper 10241-25.

Eliezer, S., Hora, H., Korn, G., Nissim, N. \& Martinez-Val, J.-M. (2016). Avalanche proton-boron fusion based on elastic nuclear collisions. Phys. Plasmas 23, 050704.

Eliezer, S., Nissim, N., Martinez Val, J.M., Mima, K. \& Hora, H. (2014). Double layer acceleration by laser radiation. Laser Part. Beams 32, 211-217.

Eliezer, S. \& Ross, D.A. (1974) A 'Cabbibo' theory for leptons and the neutrino masses. Phys. Rev. D 10, 3088.

Fan, Z., Liu, Y., Liu, B., Yu, C., Lan, K. \& Liu, J. (2017). Nonequilibrium between ions and electrons inside hot spots from National Ignition Facility experiments. MRE 2, 3-8.

Földes, I.B., Bakos, J.S., Gal, K., Juhasz, Y., Kedves, M.A., Koscis, G., Szatmari, S. \& Veres, G. (2000). Properties of high Harmonics generation by UV laser pulses on solid surfaces. Laser Phys. 10, 264.

Fortov, V.E. \& Iakubov, T. (1998). Physics of Nonideal Plasmas. Singapore: World Scientific.

Fujioka, S., Zhang, Z., Ishihara, K., Shigemori, K., HironaKa, Y., JohZaki, T., Sunahara, A., Yamamoto, N., NaKashima, H., Watanabe, T., Shiraga, H., Nishimura, H. \& Azechi, H. (2013). Kilotesla magnetic field due to a capacitor-coil target driven by high power laser. Nat. Sci. Rep. 3, 1170-1176.

Gaul, E. (2017). Discussion SPIE Prague 24 April 2017.

Giuffrida, L., Scuderi, V., Cirrone, P., Margarone, D., Picciotto, A., Velyhan, A., Peringa, G., Miluzzo, G., Krasa, J., Dostal, J., KuCHARIK, M. \& Korn, G. (2017). Inertial Fusion Science and Applications 2017, Conference abstracts, p. 92.

Hammons, T., Victor, T.J., Lesale, F., Uecker, K., Hausler, M., RetZmann, D., Staschus, K. \& LanG, S. (2012). Proceedings of the IEEE 100, No. 2, 360 DOI: 10.1109/JPROC.2011.2152310.

Hinkel, D.E., Berzak-Hopkins, L.F., Jones, O.S., Callahan, D.A. \& HurricAne, E.A. (2016). High foot implosions in larer hohlraums filled with an immediate gas fill density. IFSA Conference Seattle September 2015, Abstracts, p. 163.

Hirsch, R.L. (1972). Inertial-electrostatic confinement of ionized fusion gases. J. Appl. Phys. 38, 4522-4534.

Hoffmann, D.H.H., Hora, H., Eliezer, S., Nissim, N., Fortov, V.E., Lalousis, P., Korn, G., Moustaizis, S., KirchHoff, G.J. \& MarTINEZ-VAL, J.-M. (2017). Nonideal plasma at elastic nuclear collisions for avalanche boron fusion. Hirscheck Conference, January.

Hohenberger, M., Chang, P.-Y., Fiksel, G., Knauer, J.P., Betti, R., Marshall, F.J., Meyerhofer, D.D., Séguin, F.H. \& Petrasso, R.D. (2012). Inertial confinement fusion implosions with imposed magnetic field compression using the OMEGA Laser. Phys. Plasmas 19, 056306. 
Hora, H. (1964). Report 6/23 Abschätzungen zur Aufheizung eines Plasma mittels Lasern (Institut für Plasmaphysik, Garching) July; Estimtions for heating of plasmas with lasers. Technical Translation 1193 (National Research Council of Canada, Ottawa, 1965).

Hora, H. (1981). Physics of Laser Driven Plasma. New York: Wiley.

Hora, H. (1988). Nuclear effects and non thermal plasmas. Nucl. Instrum. Meth. A271, 117.

Hora, H. (2003). Skin-depth theory explaining anomalous picosecond-terawatt laser plasma interaction II. Czech. J. Phys. 53, 199-217.

Hora, H. (2009). Laser fusion with nonlinear force driven plasma blocks: thresholds and dielectric effects. Laser Part. Beams 29, 207-222.

HorA, H. (2013). Extraordinary strong jump of increasing laser fusion gains experienced at volume ignition for combination with NIF experiments Laser Part. Beams 31, 230-232.

Hora, H. (2014). arXiv 1412.4190.

HorA, H. (2015). SPIE NEWSROOM 10.1117/2.1201506.005965.

Hora, H. (2016). Laser Plasma Physics. 2nd edn. Bellingham, WA: SPIE Books.

Hora, H., Azechi, H., Kitagawa, K., Mima, K., Murakami, M., Nakai, S., Nishihara, K., Takabe, H., Yamanaka, C., Yamanaka, M. \& YAMANAKA, T. (1998). Measured laser fusion gains reproduced by self-similar volume compression and volume ignition for NIF conditions. J. Plasma Phys. 60, 743.

Hora, H., Badziak, J., Boody, F.P., Höpfl, R., Jungwirth, K., Kralikowa, B., Kraska, J., Laska, L., Parys, P., Perina, V., Pfeifer, M., Rohlena, K., Skala, J., Ullschmied, J., Wolowski, J. \& Woryna, E. (2002). Effects of ps and ns laser pulses for giant ion source. Opt. Commun. 207, 333-337.

Hora, H., Badziak, J., Read, M.N., Li, Y.-T., Liang, T.-J., Liu, H., Sheng, Z.-M., Zhang, J., Osman, F., Miley, G.H., Zhang, W., He, X., Peng, H., Osman, F., Glowacz, S., Jablonski, S., WolowSKi, S., Skladanowski, Z., Jungwirth, K., Rohlena, K. \& UlLschmied, J. (2007). Fast ignition by laser driven beams of very high intensity. Phys. Plasmas 14, 072701/1-7.

Hora, H., Eliezer, S., Kirchhoff, G.J., Korn, G., Lalaousis, P., Miley, G.H. \& Moustaizis, S. (2017a). Extreme laser pulses for possible development of boron fusion power reactors for clean and lasting energy. SPIE Conference Proceedings 10242 paper 10241-14.

Hora, H., Eliezer, S., Nissim, N., Xu, Y. \& Lalousis, P. (2017b). Non-thermal laser driven plasma-blocks for proton boron avalanche fusion as direct drive option. MRE 2, 177-189.

Hora, H., Eiezer, S., WANG, J., Korn, G., Nissim, N., Xu, Y., LalouSis, P., Kirchhoff, G. \& Miley, G.H. (2017c). Laser Boron Fusion Reactor with Picosecond Petawatt Block Ignition, IEEE Transactions of Plasma Science (SOFE conference proceedings).

Hora, H. \& KirchHoff, G.J. (2015). PCT/EP2014/003281 Patent, published as World Patent WO 2-15/144190 A1, priority 23 March 2014.

Hora, H. \& KirchofF, G.J. (2017). US Patent Application 20170125129A1, May 4, National Phase of PCT.

Hora, H., Korn, G., Giuffrida, L., Marganoone, D., Picciotto, A., Krasa, J., Jungwirth, K., Ullschmied, J., Lalouais, P., Eliezer, S., Miley, G.H., Moustaizis, S. \& Mourou, G. (2015a) Fusion energy using avalanche increased boron reactions for block ignition by ultrahigh power picosecond laser pulses. Laser Part. Beams. 33, 607-619.
Hora, H., Lalousis, P. \& Eliezer, S. (1984). Analysis of the inverted double layers in nonlinear force produced cavitons at laserplasma interaction. Phys. Rev. Lett. 53, 1650-1652.

Hora, H., Lalousis, P., Giuffrida, L., Margarone, D., Korn, G., Eliezer, S., MileYi, G.H., Moustaizis, S. \& Mourou, G. $(2015 b)$. Petawatt laser pulses for proton-boron high gain fusion with avalanche reactions excluding problems of nuclear radiation. SPIE Proc. 9515, 951518/1-15.

Hora, H., Lalousis, P. \& Moustaizis, S. (2014). Fiber ICAN laser with exawatt-picosecond pulses for fusion without nuclear radiation problems. Laser Part. Beams. 32, 63-68.

Hora, H., Lalousis, P., Moustaizis, S., Földes, I., Miley, G.H., Yang, X., He, X.T., Eliezer, S. \& Martinez-Val, J.-M. (2012). Shock Studies in Nonlinear Force Driven Laser Fusion with Ultrahigh Plasma Block Acceleration. IAEA Proc. Fusion Energy, San Diego October 2012. Paper IFE/P6-03, 8 pages (IAEA Vienna 2013). http://www-naweb.iaea.org/napc/ physics / FEC/FEC2012/papers /27_IFEP603.pdf

Hora, H., Malekynia, B., Ghoranneviss, M., Miley, G.H. \& He, X. (2008). Twenty times lower ignition thresholds for laser driven fusion using collective effects and the inhibition factor. Appl. Phys. Lett. 93, 011101/1-3.

Hora, H., Miley, G.H., Azizi, N., Malekynia, B., Ghoranneviss, M. \& HE, X.T. (2009). Nonlinear Force Driven Plasma Blocks Igniting Solid Density Hydrogen Boron: Laser Fusion Energy without Radioactivity. Laser Part. Beams 27, 491-496.

Hora, H., Miley, G.H., Ghorannviss, M., Malekynia, H., Azizi, N. \& HE, X-T. (2010). Fusion energy without radioactivity: laser ignition of solid hydrogen-boron(11) fuel. Energy Environ. Sci. 3, 479-486.

Hurricane, O.A., Callahan, D.A., Casey, D.T., Celliers, P.M., Cerjan, C., Dewald, E.L., Dittrich, T.R., Döppner, T., Hinkel, D.E., Berzak Hopkins, L.F., Kline, J.L., Le PaPe, S., Ma, T., Macphee, A.G., Milovich, J.L., PaK, A., Park, H.-S., Patel, P.K., Remington, B.A., Salmonson, J.D., Springer, P.T. \& Tommasini, R. (2014). Fuel gain exceeding unity in an inertial confined fusion implosion. Nature 506, 343-348.

Kanngiesser, K.W., Huang, D.H. \& LiPs, H. (1994). Highvoltage direct current transmission - Systems and Planning. Siemens monographs Munich, EV HA 7.

Labaune, C., Deprierraux, S., Goyon, S., Loisel, C., Yahia, G. \& RAFELSKI, J. (2013). Fusion reactions initiated by laser accelerated particle beams in laser produced plasmas. Nat. Commun. $\mathbf{4}$, 2506.

Lalousis, P. \& Hora, H. (1983). First direct electron and ion fluid computation of high electrostatic fields in dense inhomogeneous plasmas with subsequent nonlinear laser interaction. Laser Part. Beams 1, 283-304.

Lalousis, P., Hora, H., Eliezer, S., Martinez-Val, J.-M., Moustaizis, S., Miley, G.H. \& Mourou, G. (2013). Shock mechanisms by ultrahigh laser accelerated plasma blocks in solid density targets for fusion. Phys. Lett. A 377, 885-888.

Lalousis, P., Hora, H. \& Moustaizis, S. (2014). Optimized boron fusion with magnetic trapping by laser driven shock initiation at nonlinear force driven ultrahigh acceleration. Laser Part. Beams 32, 409

Lawrence, V.F. (1978). Momentum Transfer of Laser Radiation to Inhomogenous Dielectrics. PhD Thesis University of New South Wales. Sydney: Australia. 
Li, M., Wang, J.X., Xu, Y.X. \& Zhu, W.J. (2017) Study of plasma pressure evolution driven by strong picosecond laser pulse. Phys. Plasmas 24, 013117.

Margarone, D., Picciotto, A., Velyhan, A., Krasa, J., Kucharik, M., Mangione, A., Szydlowsky, A., Malinowska, A., Bertuccio, G., Shi, Y., Crivellari, M., Ullschmied, J., Bellutti, P. \& Korn, G. (2015). Advanced scheme for high-yield laser driven nuclear reactions. Plasma Phys. Control. Fusion 57, 014030 .

Meglich, B \& Morwood, D., Jr. (1988). Editorial: Aneutronic Power Princeton 1987. Nuclear Instruments and Methods A271, p. vii-viii.

Miley, G.H. \& K. Murali (2015) Inertial Electrostatic confinement (IEC). Heidelberg: Springer.

Norreys, P.A., Fews, A.P., Beg, F.N., Bell, A.R., Dangor, A.E., Lee, P., Nelson, M.B., Schmidt, H., Tatarakis, M. \& Cable, M.D. (1998). Neutron production from picosecond laser irradiation of deuterated targets at intensities of $10^{19} \mathrm{Wcm}^{-2}$. Phys. Control. Fusion 40, 175-182.

NucKolls, J.H. Contributions to the Genesis and Progress of UCF (2007) In Inertial Confinement Nuclear Fusion: A historical Approach by its Pioneers (Guillermo Velarde and Natividad Carbintero-Santamarai eds) Foxwell \& Davies (UK) p.493-507 ISBN 1-805868-10. 2007.

Oliphant, M.L.E. \& Rutherford, L. (1933). Experiments on the transmutations of elements by protons. Proc. R. Soc. Lond. A 141, 259-281.

Picciotto, A., Margarone, D., Velyhan, A., Bellini, P., Krasa, J., Szydlowski, A., Bertuccio, G., Shi, Y., Margarone, A., Prokupek, J., Malinowska, A., Krouski, E., Ullschmied, J., Laska, L., KuCHARIK, M. \& KorN, G. (2014). Boron-proton nuclear-fusion enhancement induced in boron-doped silicon targets by low contrast pulsed laser. Phys. Rev. X 4, 031030.

Rostoker, N. \& Binderbauer, M.W. (1996). US-Patent 6,664,740 B-2.

Rostoker, N., Binderbauer, M.W. \& Monkhorst, H.J. (1997). Colliding beam fusion reactor. Science 278, 1419-1422.
Sakharov, A.D. (1982). Collected Scientific Works. NewYork and Basel: Marcel and Dekker, 1983, (see Laser and Particle Beams 18, 151 2000).

SAntos, J.J., Bailly-Gandvoux, M., Giuffrida, L., Forestercolleoni, P., Fujooka, S., Zang, Z., Korneev, PH, Boullaud, R., Dor And, S., Batani, D., Chervrrot, M., Cross, J., Crowston, R., Dubois, J.-L., Gazave, J., Greofri, G., D'humieres, E., Hulin, S., Ishihara, K., KoJima, S., Loyez, E., Marques, J.-R., Nicolai, PH., Peyrusse, O., Poye, A., Raffestin, D., Ribolzi, J., Roth, M., Schaumann, G., Serred, F., Tikhonchuk, V.T., Vacar, PH., Woolsley, N. (2015). Laser-driven platform for generation and characterization of strong quasi-static magnetic fields. New J. Phys. 17, 083061/1-16.

SAuERBrey, R. (1996). Acceleration of femtosecond laser produced plasmas. Phys. Plasmas 3, 4712-4716.

TabaK, M., Hammer, J., Glinsky, M.N., Kruer, W.L., Wilks, S.C., Woodworth, J., Campbell, E.M., Perry, M.D. \& Mason, R.J. (1994). Ignition and high gain with ultrapowerful lasers. Phys. Plasmas 1, 1626-1634.

TAhiR, N.A. \& Hoffmann, D.H.H. (1997). Development of advanced inertial fusion targets. Laser Part. Beams 15, 575-587.

Tikhonchuk, V., Baylly-Grandvaux, M., Santos, J.J. \& Poye, A. (2017). Quai-stationary magnetic field generation with capacitorcoil targets. Inertial Fusion Science and Applications 2017, Conference abstracts p. 81

Xu, Y., WAnG, J.X., QI, M., Li, Y., XING, Y. \& Long, L. (2016). Improving the quality of proton beams via double targets driven by an intense circularly polarized laser pulse. AIP Adv. 6, 105304.

Xu, Y., WANG, J.X., QI, M., LI, Y., XING, Y., LonG, L. \& ZHU, W. (2017). Plasma block acceleration via double targets driven by an ultraintense circulatory polarized laser pulse. Phys. Plasmas 24, 033108.

Zhang, M., He, J.T., Chen, D.B., Li, Z.H., Zhang, Y., Wang, L., Feng, B.L., Zhang, D.F., TANG, X.W. \& Zhang, J. (1998). Effects of a prepulse on $\gamma$-ray radiation produced by a femtosecond laser with only 5-mJ energy. Phys. Rev. E 57, 3746-3748. 